Crawford, K. \& Abraham, E. P. (1957). J. gen. Microbiol. 16, 604-613

\title{
The Synergistic Action of Cephalosporin G and Benzyl- penicillin against a Penicillinase-producing Strain of Staphylococcus aureus
}

\author{
BY KATHLEEN CRAWFORD AND E. P. ABRAHAM \\ Sir William Dunn School of Pathology, University of Oxford
}

SUMMARY: Cephalosporin C, a hydrophilic, penicillin-like antibiotic which is insensitive to penicillinase, is much less active than benzylpenicillin against the Oxford strain of Staphylococcus aureus but much more active than benzylpenicillin against a penicillinase-producing strain of $S$. aureus (D3R). When used together, cephalosporin $C$ and benzylpenicillin act synergistically against $S$. aureus strain D3R in vitro, presumably because cephalosporin $\mathrm{C}$ is a competitive inhibitor of penicillinase. When grown under certain conditions in the presence of cephalosporin $\mathrm{C}$, benzylpenicillin, or bacitracin, the Oxford staphylococcus gives rise during a single culture to a resistant bacterial population consisting largely or partly of Gram-negative bacilli. In the presence of bacitracin a similar change also occurs with $S$. aureus strain D3R.

Two hydrophilic antibiotics, cephalosporin $\mathbf{N}$ and cephalosporin $\mathbf{C}$, have been isolated from the culture fluid of a species of Cephalosporium (Abraham, Newton \& Hale, 1954; Newton \& Abraham, 1956). Cephalosporin $\mathrm{N}$ is a new type of penicillin with a side-chain derived from $D-\alpha$-aminoadipic acid (Newton \& Abraham, 1954), and appears to be identical with synnematin B (Abraham et al. 1955). Cephalosporin $\mathrm{C}$ is chemically related to cephalosporin $\mathrm{N}$, but is not a true penicillin (Abraham \& Newton, 1956a). It is not sensitive to penicillinase, but is a competitive inhibitor of the action of this enzyme on penicillin (Abraham \& Newton, 1956b), and it induces the formation of penicillinase by Bacillus cereus (Pollock, 1957). It has a low antibacterial activity, but no significant toxicity to mice, and it affords protection to mice infected with Streptococcus pyogenes or with a penicillin-resistant strain of Staphylococcus aureus (Florey, 1955, 1957).

The finding that cephalosporin $\mathrm{C}$ was a competitive inhibitor of penicillinase raised the question whether it would act synergistically with penicillin against penicillin-resistant staphylococci which produce this enzyme. The work described in the present paper was begun with the object of obtaining an answer to this question. During the work it was observed that staphylococci underwent profound morphological and biochemical changes when grown under certain conditions in media containing cephalosporin $\mathrm{C}$, benzylpenicillin, or bacitracin.

\section{METHODS}

Materials. Benzylpenicillin (1650 units/mg., Glaxo Laboratories Ltd.) and cephalosporin C (Newton \& Abraham, 1956) were used as the crystalline sodium salts. The bacitracin was a commercial sample (A/S Apothekernes 
Laboratorium, Oslo) with an activity of 65 units/mg.; it consisted mainly of bacitracin A together with some bacitracin B. Crystalline micrococcin (Heatley \& Doery, 1951; Abraham, Heatley, Brookes, Fuller \& Walker, 1956) was kindly provided by Dr N. G. Heatley.

A penicillin-sensitive strain of Staphylococcus aureus (Oxford strain, NCTC 6571) and a resistant strain which produced penicillinase (strain D3R, phage type $6 / 47^{+}$; kindly provided by Dr Mary Barber) were used as test organisms. The penicillin-resistant strain D3R appeared not to produce a significant amount of an enzyme able to destroy cephalosporin C (cf. Abraham $\&$ Newton, 1956 b). The culture medium used was in most cases a tryptic heart digest broth (TMB; Barnes, 1955). One batch of medium was used in many experiments.

\section{Measurements of antibacterial activity}

(1) Two-fold serial dilution tests were carried out at $37^{\circ}$ in the conventional manner, the lowest concentration of antibiotic which prevented visible growth for different periods of time being recorded. In tests with mixtures of antibiotics the concentration of each component of the mixture was varied (in two-fold steps) independently.

(2) Aerated cultures from large inocula (which gave about $10^{7}$ cocci $/ \mathrm{ml}$.) were grown in inverted T-tubes of the type devised by Dr J. Monod (see Kay \& Fildes, 1950). The tubes were immersed in a water bath at $37^{\circ}$ and attached to an apparatus by which they were rocked 44 times $/ \mathrm{min}$. Changes in the opacity were measured in a 'Spekker' absorptiometer (Adam Hilger Ltd.), a neutral grey filter (H508) being used. The inoculum was taken from an overnight stagnant culture in TMB. The antibiotic was usually added about $1.5 \mathrm{hr}$. after inoculation, when growth had just entered the logarithmic phase.

\section{RESULTS}

\section{Activity of cephalosporin $C$ and benzylpenicillin against different strains of Staphylococcus aureus}

Serial dilution tests. Table 1 shows the effect of cephalosporin $\mathbf{C}$ alone, benzylpenicillin alone, and mixtures of the two on the growth of the penicillinsensitive (Oxford) strain of Staphylococcus aureus. It is evident that benzylpenicillin was more than 1000 times as active as cephalosporin $\mathrm{C}$ against this

\section{Table 1. Inhibition of growth of Staphylococcus aureus (Oxford strain)}

The inoculum was $1 \mathrm{drop} / \mathrm{ml}$. of an overnight TMB culture diluted 1/1000. The bracketed figures show the concentrations for various inhibitory mixtures of benzylpenicillin (upper values) and cephalosporin $C$ (lower values). In general, growth occurred when the concentration of either component of an inhibitory mixture was halved.

\section{Antibiotic}

Benzylpenicillin alone Cephalosporin $\mathbf{C}$ alone Benzylpenicillin $+$ Cephalosporin $\mathrm{C}$
Concentration $(\mu \mathrm{g} . / \mathrm{ml}$.) inhibitory to growth for

\begin{tabular}{|c|c|c|c|}
\hline 1 day & 2 days & 3 days & 6 days \\
\hline 0.015 & 0.015 & 0.03 & 0.06 \\
\hline 50 & 100 & 100 & 100 \\
\hline 0.015$)$ & $0.015)$ & $0.015)$ & 0.015 \\
\hline $12 \cdot 5$ & 50 & 50 & 50 \\
\hline
\end{tabular}


organism under the conditions used; that the concentration of either compound required to inhibit growth for 6 days was not very much greater than that required to inhibit growth for 1 day; and that the activity of a mixture of the two compounds was not significantly greater than would be predicted on the assumption that their effect together was purely additive.

Tables 2 and 3 show the results of two similar experiments in which the penicillinase-producing Staphylococcus aureus (D3R) was the test organism. In one case (Table 3) the inoculum was 100 times as large as in the other. The larger inoculum gave about $10^{6} \mathrm{cocci} / \mathrm{ml}$. Benzylpenicillin was much more active against the smaller inoculum than against the larger one, and with both inocula it was much more effective in suppressing growth for 1 day than for 5 or 6 days. In contrast, the activity of cephalosporin $\mathrm{C}$ varied little with the size of the inoculum or with the time at which the end-point was recorded. With the small inoculum cephalosporin $\mathrm{C}$ appeared to be 5 times as active as benzylpenicillin after 6 days and with the large inoculum it appeared to be $\mathbf{2 0}$ times as active after $\mathbf{5}$ days. In both cases the activities of mixtures of cephalosporin $\mathbf{C}$ and benzylpenicillin were considerably greater than the sum of the activities of their components.

\section{Table 2. Inhibition of growth of Staphylococcus aureus strain D3R (penicillinase producer)}

The inoculum was $1 \mathrm{drop} / \mathrm{ml}$. of an overnight TMB culture diluted 1/1000. The bracketed figures show the concentrations for various inhibitory mixtures of benzylpenicillin (upper values) and cephalosporin $\mathrm{C}$ (lower values). Readings after 6 days showed that growth occurred when the concentration of either component of an inhibitory mixture was halved.

\begin{tabular}{|c|c|c|c|c|}
\hline Antibiotic & 1 day & 2 days & 3 days & 6 days \\
\hline Benzylpenicillin alone & 0.5 & 4 & 16 & 500 \\
\hline Cephalosporin $\mathrm{C}$ & 100 & 100 & 100 & 100 \\
\hline $\left.\begin{array}{c}\text { Benzylpenicillin } \\
+\end{array}\right\}$ & - & 2 & - & 4 \\
\hline Cephalosporin C & - & $\left.\begin{array}{c}12 \cdot 5 \\
2 \\
6 \cdot 25\end{array}\right\}$ & - & $\left.\begin{array}{c}12 \cdot 5 \\
16 \\
6 \cdot 2\end{array}\right\}$ \\
\hline
\end{tabular}

The organisms which grew up, in these tests, in the presence of the antibiotics appeared to be morphologically indistinguishable from normal staphylococci, except that they tended to be somewhat larger.

Changes in opacity of aerated cultures from large inocula. The results of a typical experiment in which cephalosporin $\mathrm{C}$ alone, benzylpenicillin alone and mixtures of the two antibiotics were added to cultures of the penicillinaseproducing strain D3R of Staphylococcus aureus growing in aerated TMB are shown in Fig. 1. Under these conditions, the addition of the antibiotic caused no appreciable change in the rate of bacterial growth for about $1 \mathrm{hr}$. but, at the 
end of that time, growth rapidly gave way to extensive lysis, leaving a culture which showed only a faint turbidity. After a period whose length depended on the initial concentration of the antibiotic, visible growth again took place.

\section{Table 3. Inhibition of growoth of Staphylococcus aureus strain D3R (penicillinase producer)}

The inoculum was $1 \mathrm{drop} / \mathrm{ml}$. of an overnight $\mathrm{TMB}$ culture diluted $1 / 10$. The bracketed figures show the concentrations for various inhibitory mixtures of benzylpenicillin (upper values) and cephalosporin $\mathrm{C}$ (lower values). Readings after 3 or 5 days showed that growth occurred when the concentration of either component of an inhibitory mixture was halved.

\section{Concentration $(\mu \mathrm{g} . / \mathrm{ml}$.) inhibitory to growth for}

\begin{tabular}{|c|c|c|c|c|}
\hline Antibiotic & 1 day & 2 days & 3 days & 5 days \\
\hline Benzylpenicillin alone & 4 & 250 & 2000 & 4000 \\
\hline Cephalosporin $\mathrm{C}$ alone & 100 & 200 & 200 & 200 \\
\hline $\left.\begin{array}{c}\text { Benzylpenicillin } \\
+\end{array}\right\}$ & $2)$ & 60 & 120 & 30 \\
\hline Cephalosporin $\mathrm{C}$ ] & 50 & $6 \cdot 2$ & $12 \cdot 5$ & $\left.\begin{array}{r}50 \\
120 \\
25\end{array}\right\}$ \\
\hline
\end{tabular}

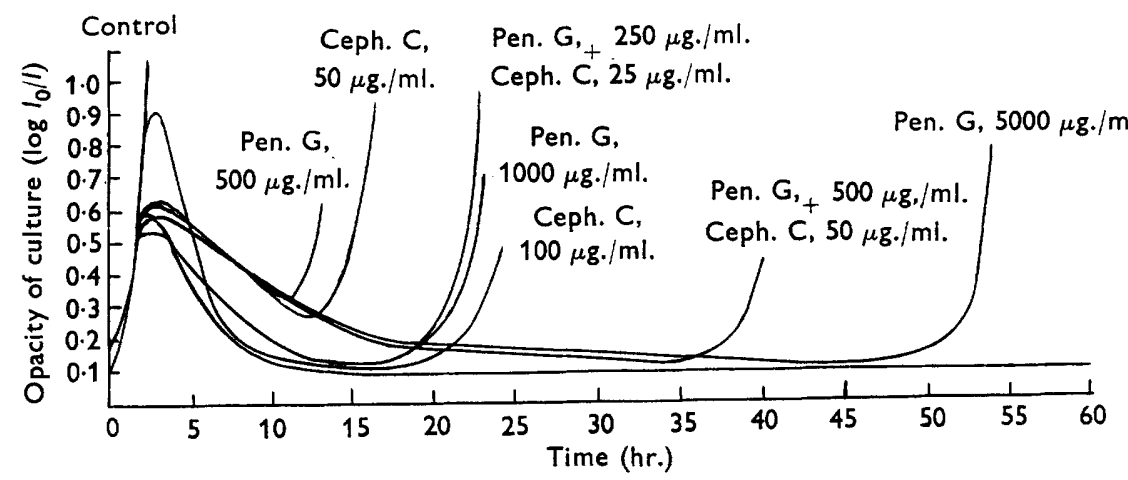

Fig. 1. Changes in the opacity of an aerated culture of Staphylococcus aureus strain D3R, on the addition of cephalosporin $\mathrm{C}$ alone, benzylpenicillin alone and mixtures of both antibiotics. $\leftarrow$ shows the times at which the antibiotics were added. Pen. $\mathrm{G}=$ benzylpenicillin; ceph. $\mathbf{C}=$ cephalosporin $\mathbf{C}$.

In terms of its ability to delay final growth, cephalosporin $\mathrm{C}$ was more than 10 times as active as benzylpenicillin against Staphylococcus aureus strain D3R. (In a similar experiment in which the medium was Difco heart infusion broth cephalosporin $\mathrm{C}$ was 40 times as active as benzylpenicillin against strain D3R.) When the two antibiotics were used together they acted synergistically. Thus, Fig. 1 shows that a mixture of $250 \mu \mathrm{g}$. benzylpenicillin $/ \mathrm{ml}$. and $25 \mu \mathrm{g}$. cephalosporin $\mathrm{C} / \mathrm{ml}$. was considerably more effective than $500 \mu \mathrm{g}$. benzylpenicillin $/ \mathrm{ml}$. alone or $50 \mu \mathrm{g}$. cephalosporin $\mathrm{C} / \mathrm{ml}$. alone, and that similar results were obtained when the substances were used in double these concentrations. With the Oxford staphylococcus the changes in opacity were similar to those shown in Fig. 1. With this organism, however, $100 \mu \mathrm{g}$. cephalosporin $\mathrm{C} / \mathrm{ml}$. or $1 \mu \mathrm{g}$. benzylpenicillin $/ \mathrm{ml}$. was necessary to delay final growth for about $35 \mathrm{hr}$. in the batch of TMB used. 


\section{Development of resistance to cephalosporin $C$ and benzylpenicillin}

Cultures obtained when Staphylococcus aureus strain D3R finally multiplied in aerated TMB containing either cephalosporin $\mathrm{C}$ or benzylpenicillin (Fig. 1) were more resistant to both antibiotics than the parent strain. Figure 2 shows the result of a typical experiment in which cocci from a culture grown in the presence of $100 \mu \mathrm{g}$. cephalosporin $\mathrm{C} / \mathrm{ml}$. were inoculated into fresh medium and $200 \mu \mathrm{g}$. cephalosporin $\mathrm{C} / \mathrm{ml}$. added. Heavy growth occurred almost as rapidly as from a control inoculum in medium which did not contain cephalosporin C. A similar phenomenon was observed with benzylpenicillin. For example, in one experiment the original D3R staphylococcus began to undergo the secondary multiplication after $40 \mathrm{hr}$. in the presence of $4 \mathrm{mg}$. benzylpenicillin $/ \mathrm{ml}$. An inoculum from the resulting culture grew up in the presence of the same concentration of benzylpenicillin after $10 \mathrm{hr}$. As measured in this way, increased resistance to cephalosporin $\mathrm{C}$ was accompanied by similar increased resistance to benzylpenicillin and vice versa. In serial dilution tests (read after 6 days and with the inoculum taken directly from the T-tube) the culture which had acquired resistance in the presence of benzylpenicillin was 16 times as resistant to this antibiotic as was the parent, whereas the culture which had acquired resistance in the presence of cephalosporin $\mathrm{C}$ was 8 times as resistant to benzylpenicillin as the parent. No evidence was obtained that an increased resistance to cephalosporin $\mathrm{C}$ was associated with the development of an ability to destroy this antibiotic. Samples of culture fluid taken at various times from the T-tubes were tested for cephalosporin $\mathrm{C}$ by the holeplate method. Some loss of activity (at least 50\%) appeared to have occurred by the time that final growth was complete. However, the grown resistant culture (Fig. 2) still contained more cephalosporin $\mathrm{C}$ than would have been required to bring about rapid lysis of the original culture. The resistance which developed under these conditions during the growth of a single culture was readily reversible. When cocci from the resistant culture were allowed to grow once in the absence of antibiotic, they gave rise to a new culture which was almost as sensitive as the parent strain.

Changes in resistance occurred in a similar manner when the benzylpenicillin-sensitive Oxford strain of Staphylococcus aureus was used; as with $S$. aureus strain D3R, there was cross-resistance to cephalosporin $\mathrm{C}$ and benzylpenicillin. With the Oxford staphylococcus, however, changes in resistance were accompanied by changes in morphology. The bacterial population which resulted from the final multiplication of the Oxford staphylococcus in aerated TMB consisted of varying proportions of Gram-positive cocci and Gram-negative bacilli (cf Briggs, Crawford, Abraham \& Gladstone, 1957).

\section{Experiments with bacitracin and micrococcin}

Bacitracin A, a polypeptide antibiotic containing sulphur in a thiazoline ring (Lockhart, Abraham \& Newton, 1955; Hausmann, Weisiger \& Craig, 1955), has been reported to resemble penicillin in its effect on the incorporation of amino acids into bacterial protein (Gale \& Folkes, 1953). Cross-resistance 
to bacitracin and penicillin has also been reported (Paine, 1951). Micrococcin is a complex antibiotic with a high percentage of sulphur and micrococcin $\mathbf{P}$, an identical or very similar substance, has been shown by Brookes, Fuller \& Walker (1957) to contain the thiazole ring system. It therefore seemed worth while to compare the effects of bacitracin and micrococcin with that of benzylpenicillin on staphylococci growing in aerated TMB.

Bacitracin. When bacitracin (final concentration $154 \mu \mathrm{g} . / \mathrm{ml}$.) was added to a growing culture of the Oxford staphylococcus or Staphylococcus aureus strain D3R the changes in opacity were very similar to those shown in Fig. 1. Growth

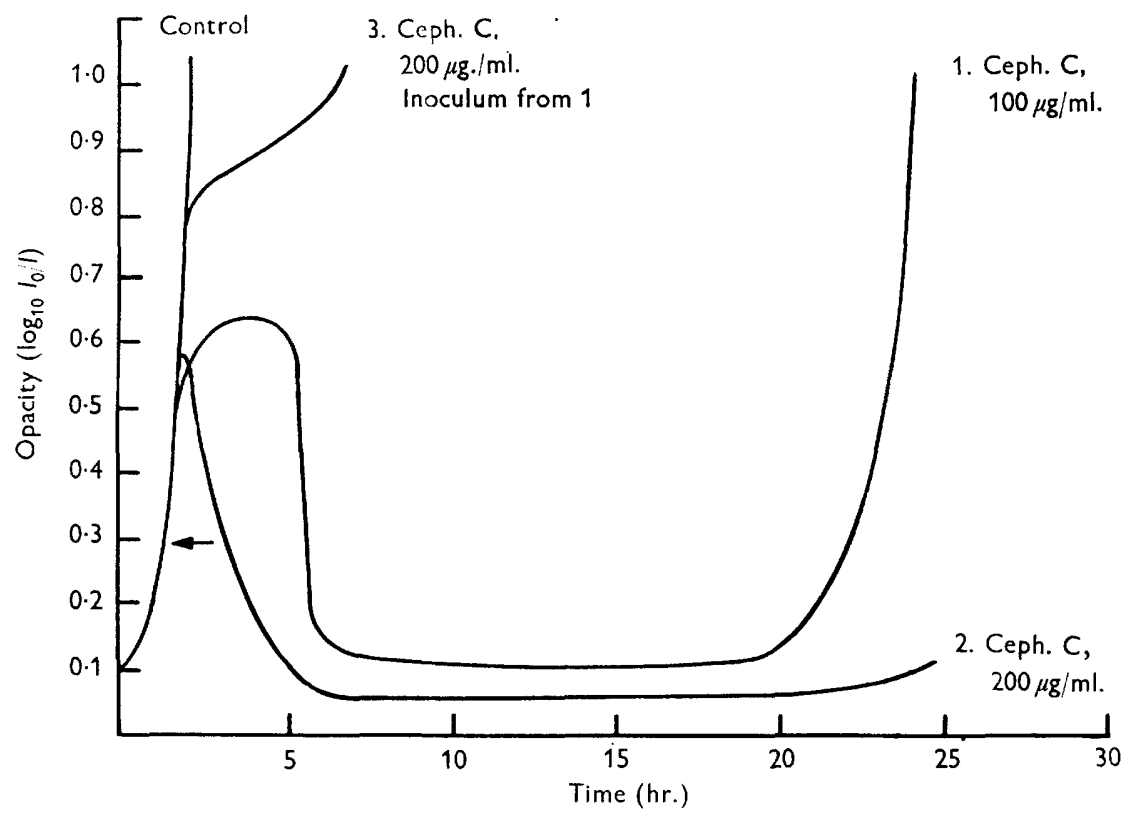

Fig. 2. Resistance acquired by Staphylococcus aureus strain D3R to cephalosporin C. $\leftarrow$ shows the point at which the antibiotic was added.

continued rapidly for about $2 \mathrm{hr}$. and was then followed by lysis. A subsequent increase in opacity began again after $30 \mathrm{hr}$. (Oxford staphylococcus) and $20 \mathrm{hr}$. (strain D3R). In both cases the final culture consisted of a mixture of apparently normal staphylococci and Gram-negative rods in approximately equal proportions and was more resistant to bacitracin than the parent. Inocula taken from the cultures which had grown once in the presence of bacitracin (referred to as culture $\mathrm{B}_{1}$ from the Oxford staphylococcus, and culture $\mathrm{B}_{\mathbf{2}}$ from strain D3R) began to undergo the secondary multiplication after 4 and $5 \mathrm{hr}$. respectively, when used in fresh medium containing the same concentration of the drug.

The increase in resistance of Staphylococcus aureus strain D3R to bacitracin was also found to be accompanied by an increase in resistance to cephalosporin $\mathrm{C}$ and benzylpenicillin. In a comparative experiment, the final increase in opacity of culture $B_{2}$ in the presence of cephalosporin $C(200 \mu \mathrm{g} . / \mathrm{ml}$.) 
occurred after $18 \mathrm{hr}$., whereas the corresponding time with strain D3R was $30 \mathrm{hr}$. In the presence of benzylpenicillin $\left(1000 \mu \mathrm{g}\right.$. $/ \mathrm{ml}$.) the time for culture $\mathbf{B}_{2}$ was $15 \mathrm{hr}$. and that for strain D3R was $20 \mathrm{hr}$.

Micrococcin. When micrococcin (final concentration $1 \mu \mathrm{g} . / \mathrm{ml}$.) was added to a growing culture of Staphylococcus aureus D3R, there was an immediate but relatively slight decrease in the growth rate for $3 \mathrm{hr}$., followed by a much larger decrease in the next $20 \mathrm{hr}$; within $40 \mathrm{hr}$. the culture appeared to be almost as dense as a control culture. No detectable lysis occurred and the organisms in the final culture had the appearance of normal staphylococci.

\section{DISCUSSION}

The effect of cephalosporin $\mathbf{C}$ on the opacity of growing cultures of staphylococci is qualitatively very similar to that of benzylpenicillin, a fact which is consistent with the suggestion that cephalosporin $\mathbf{C}$ is a penicillin-like substance. The relatively low activity of cephalosporin $\mathbf{C}$ against penicillinsensitive staphylococci may be associated with an inability of the organisms to concentrate this compound in the way that they concentrate benzylpenicillin. A similar suggestion was made to account for the finding that the activity of cephalosporin $\mathbf{N}$ against many Gram-positive organisms was much lower than that of the common penicillins (Abraham, Newton, Crawford, Burton \& Hale, 1953). Pollock (1957) has shown that the relative 'affinities' of benzylpenicillin, cephalosporin $\mathbf{N}$ and cephalosporin $\mathbf{C}$ for the 'penicillin-binding component' in Bacillus cereus (as measured by their ability to inhibit fixation of ${ }^{35} \mathrm{~S}$ from ${ }^{35} \mathrm{~S}$-labelled benzylpenicillin) bear some relationship to their relative activities as antibiotics against Gram-positive organisms. However, it is not yet possible to draw a physico-chemical picture of the processes on which these 'affinities' depend.

In contrast to the situation with the penicillin-sensitive staphylococcus, the activity of cephalosporin $\mathrm{C}$ in suppressing visible growth of a penicillinaseproducing strain of staphylococcus for a prolonged period was much greater than that of benzylpenicillin. This fact is clearly related to the stability of cephalosporin $\mathrm{C}$ to the action of penicillinase. In serial dilution tests the apparent activity of benzylpenicillin was less with a large inoculum than with a small one, and it decreased greatly as the times at which the test was read was extended from 1 to 6 days. The activity of cephalosporin C, on the other hand, varied little with the size of the inoculum or with the time.

In serial dilution tests with the Oxford staphylococcus, the total activity of a mixture of cephalosporin $\mathrm{C}$ and benzylpenicillin was not greatly different from the sum of the activities of the separate components. This simple additive effect would be expected if both antibiotics had a similar mode of action, the difference in their separate specific activities being due to a difference in their ability to combine with the sensitive centre in the cell.

In serial dilution tests with the penicillinase-producing staphylococcus strain D3R, the activity of a mixture of cephalosporin $\mathrm{C}$ and benzylpenicillin was much greater than the sum of the separate activities of the components in 
suppressing growth for 5-6 days. The figures given in Table 2 show that the activities of mixtures were from 7 to 10 times as great as would have been expected on the assumption that a solution to which had been added $x$ units of cephalosporin $\mathrm{C}$ activity and $y$ units of benzylpenicillin activity would contain $x+y$ units. The corresponding values obtained from an experiment in which the inoculum was 100 times as large (Table 3 ) vary from 4 to 7 . With aerated cultures in T-tubes, in which even larger inocula were used, the activity of a mixture of the antibiotics in suppressing final growth for 1-2 days was nearly twice as great as the sum of the activities of its components (Fig. 1).

We have applied the term synergism to the increased activity of mixtures of cephalosporin $\mathrm{C}$ and benzylpenicillin. This word has come into use as a descriptive term for the phenomenon of two drugs together being more effective than either alone. The phenomenon may have various underlying mechanisms and it has been measured in different ways. Eagle \& Saz (1955) have maintained that the term synergism should not be used when two drugs act independently on different members of a population, and that it should be restricted to those cases in which one drug augments the action of another on individual cells. It seems more convenient, however, to give it a broader meaning.

There can be little doubt that part, at least, of the synergistic action of cephalosporin C and benzylpenicillin on Staphylococcus aureus strain D3R is due to the fact that cephalosporin $\mathbf{C}$ is a competitive inhibitor of penicillinase. The two antibiotics showed no significant synergistic action on the Oxford staphylococcus, which does not produce penicillinase. Although the relative affinity of cephalosporin $\mathrm{C}$ and benzylpenicillin for penicillinase is known (Abraham \& Newton, 1956b) it is not sufficient, without knowledge of other variables, for a quantitative prediction to be made of the combined effect of the two drugs in serial dilution tests. Nevertheless, some of the semi-quantitative aspects of the experimental findings seem to be understandable.

In the two serial dilution tests with Staphylococcus aureus strain D3R it appeared that synergism was greater with a small inoculum than with a large one and greater when the test was read after 5-6 days than when it was read after 1 or 2 days. The following considerations suggest that the synergistic effects, as measured here, will be greatest when the amount of penicillinase formed in the culture is neither very large nor very small. Suppose that growth does not occur before the concentration of benzylpenicillin has fallen to a given value. With a very big inoculum, producing much penicillinase, the destruction of benzylpenicillin will be rapid and a large amount of this drug alone will be necessary to suppress growth for a prolonged period. The inhibitory concentration of cephalosporin $\mathrm{C}$ alone will be relatively much smaller. The addition of subinhibitory amounts of cephalosporin $\mathbf{C}$ may therefore have only a minor effect on the rate of destruction of most of the benzylpenicillin, since the two compete for the penicillinase. On the other hand, if the inoculum were so small that very little penicillinase was produced, or if the tests were read as early as possible, so that the enzyme had relatively little time to act, the inhibitory concentration of benzylpenicillin alone might begin to approach 
the theoretical value that it would have if no destruction by penicillinase occurred. Under such conditions the maximum synergistic effect that would be possible might not be very great. Whether a significant effect will be demonstrable under some conditions in vivo remains uncertain.

Both the Oxford staphylococcus and Staphylococcus aureus strain D3R became more resistant to cephalosporin $\mathrm{C}$ and benzylpenicillin when grown in aerated cultures in the presence of these antibiotics. 'The finding that an increase in resistance to one substance was accompanied by an increase in resistance to the other is consistent with the view that they have similar modes of action.

The Oxford staphylococcus gave rise, in the presence of cephalosporin $\mathrm{C}$, benzylpenicillin, or bacitracin to a Gram-negative bacillus. Klimek, Cavallito \& Bailey (1948) reported that pleomorphic Gram-negative organisms were obtained from a strain of Staphylococcus aureus which had been transferred 64 times in medium containing increasing amounts of penicillin. The change encountered during the present work, however, is remarkable in that it occurred during the growth of a single culture. The ability to change in this way is not confined to one strain of staphylococcus for Gram-negative organisms were obtained with equal facility when $S$. aureus strain D3R was grown in the presence of bacitracin. Briggs et al. (1957) have made a further study of the bacilli formed from the Oxford staphylococcus in the presence of penicillin.

We wish to thank Miss Mary Read and Mrs P. Curtiss for technical assistance. We are grateful to Dr G. G. F. Newton and to the staff of the Medical Research Council's Antibiotic Research Station (Clevedon, Somerset) for samples of Cephalosporin C.

\section{REFERENCES}

Abraham, E. P., Heatley, N. G., Brookes, P., Fuller, A. T. \& Walker, J. (1956). Probable identity of an antibiotic produced by a spore-bearing bacillus of the B. pumilus group with micrococcin. Nature, Lond. 178, 44.

Abraham, E. P. \& Newton, G. G. F. (1956a). Experiments on the degradation of cephalosporin C. Biochem. J. $62,658$.

Abraham, E. P. \& Newton, G. G. F. (1956b). A comparison of the action of penicillinase on benzylpenicillin and cephalosporin $\mathrm{N}$ and the competitive inhibition of penicillinase by cephalosporin C. Biochem. J. 63, 628 .

Abraham, E. P., Newton, G. G. F., Crawford, K., Burton, H. S. \& Hale, C. W. (1953). Cephalosporin $\mathrm{N}$ : a new type of penicillin. Nature, Lond. 171, 343.

Arraham, E. P., Newton, G. G. F. \& Hale, C. W. (1954). Purification and some properties of cephalosporin N, a new penicillin. Biochem. J. 58, 94.

Abraham, E. P., Olson, B. H., Newton, G. G. F., Schurmans, D. M., Schenck, J. R., Fisher, M. W., Hargie, M. P. \& Fusari, S. A. (1955). Identity of cephalosporin $\mathrm{N}$ and synnematin B. Nature, Lond. 176, 551.

BARNES, E. M. (1955). Bacterial growth on heart digest media in relation to the trypsin preparation used. J. appl. Bact. 18, 45.

Briggs, S., Crawford, K., Abraham, E. P. \& Gladstone, G. P. (1957). Some properties of Gram-negative bacilli obtained from a strain of Staphylococcus aureus in the presence of benzylpenicillin. J. gen. Microbiol. 16, 614 .

Brookes, P., Fuller, A. T. \& Walker, J. (1957). The chemistry of micrococcin P. Part 1. J. chem. Soc. p. 689.

EAgle, H. \& SAz, A. K. (1955). Antibiotics. Annu. Rev. Microbiol. 9, 173. 
Florey, H. W. (1955). Antibiotic products of a versatile fungus. Ann. intern. Med. 43, 480 .

Florey, H. W. (1957). The medical aspects of the development of resistance to antibiotics. First European Symposium on Antibiotics, Milan. G. Microb. (in the Press).

Gale, E. F. \& Folkes, J. P. (1953). The assimilation of amino acids by bacteria. Biochem. J. 55, 721.

Hausmann, W., Weisiger, J. R. \& Craig, L. C. (1955). Partial hydrolysis studies with bacitracin. J. Amer. chem. Soc. 77, 721.

Heatley, N. G. \& Doery, H. M. (1951). The preparation and some properties of . purified micrococcin. Biochem. J. 50, 247.

KAY, D. \& Frldes, P. (1950). The calcium requirements of a typhoid bacteriophage. Brit. J. exp. Path. 31, 338.

Klimek, J. W., Cavaluito, C. J. \& Bailey, J. H. (1948). Induced resistance of Staphylococcus aureus to various antibiotics. J. Bact. 55, 139.

Lockhart, I. M., Abraham, E. P. \& Newton, G. G. F. (1955). The $N$-terminal and sulphur-containing residues of bacitracin A. Biochem. J. 61, 534.

Newton, G. G. F. \& Abraham, E. P. (1954). Degradation, structure and some derivatives of cephalosporin N. Biochem. J. 58, 103.

Newton, G. G. F. \& Abraham, E. P. (1956). Isolation of cephalosporin C, a penicillin-like antibiotic containing $\mathrm{D}$ - $\alpha$-aminoadipic acid. Biochem. J. 62, 651 .

PaIne, T. F. (1951). The similarity in action of bacitracin and penicillin on the staphylococcus. J. Bact. 61, 259.

Pollock, M. R. (1957). The activity and specificity of inducers of penicillinase production in Bacillus cereus NRRL 569. Biochem. J. (in the Press).

(Received 16 November 1956) 